\title{
A Sub-100 mg Electromagnetically Driven Insect-inspired Flapping-wing Micro Robot Capable of Liftoff and Control Torques Modulation
}

\author{
Chenyang Wang, Weiping Zhang*, Yang Zou, Ran Meng, Jiaxin Zhao, Mingchen Wei \\ National Key Laboratory of Science and Technology on Micro/Nano Fabrication, School of Electronic Information and \\ Electrical Engineering, Shanghai Jiao Tong University, Shanghai 200240, China
}

\begin{abstract}
Inspired by the unique, agile and efficient flapping flight of insects, we present a novel sub-100 mg, electromagnetically driven, tailless, flapping-wing micro robot. This robot utilizes two optimized electromagnetic actuators placed back to back to drive two wings separately, then kinematics of each wing can be independently controlled, which gives the robot the ability to generate all three control torques of pitch, roll and yaw for steering. To quantify the performance of the robot, a simplified aerodynamic model is used to estimate the generated lift and torques, and two customized test platforms for lift and torque measurement are built for this robot. The mean lift generated by the robot is measured to be proportional to the square of the input voltage amplitude. The three control torques are measured to be respectively proportional to three decoupled parameters of the control voltages, therefore the modulation of three control torques for the robot is independent, which is helpful for the further controlled flight. All these measured results fit well with the calculated results of the aerodynamic model. Furthermore, with a total weight of $96 \mathrm{mg}$ and a wingspan of $3.5 \mathrm{~cm}$, this robot can generate sufficient lift to take off.
\end{abstract}

Keywords: insect-inspired, insect-scale, micro robot, FMAV, electromagnetic actuation

Copyright $\odot$ The author(s) 2020 .

\section{Introduction}

In order to imitate the efficient and agile tailless flight of insects. Researchers have been creating insect inspired Flapping-wing Micro Air Vehicles (FMAV) over the past decades, and several motor driven FMAVs are reported to achieve controlled flight ${ }^{[1-7]}$. However, limited by the size of the motor and the complex transmission, these FMAVs weigh around $20 \mathrm{~g}$ with the size close to the human palm, which is far more than real insects. To be more similar to insects, some researchers have been focusing on the research of the insect-scale tailless FMAVs and several studies have successfully demonstrated liftoff ${ }^{[8-12]}$. Unlike the rotary displacement output of the motor, these insect-scale FMAVs use reciprocating actuators to mimic the contraction of insect muscles, which can realize the flapping of the wings through a simpler transmission mechanism ${ }^{[8,10-12]}$ or even without transmission ${ }^{[9]}$. However, the simpler mechanical structures increases the difficulty of the control torques modulation for steering and the follow-up controlled flight. Inspired by the flight mechan- isms and control strategies of hovering insects, a novel control method called Split-Cycle Constant-Period Frequency Modulation (SCCPFM) was proposed by Oppenheimer ${ }^{[13]}$, providing a high level of control input for insect- scale tailless FMAVs with independently actuated and passive rotated wings. By using SCCPFM, CMU, Harvard University, and Purdue University successfully demonstrated all three control torques modulation of their designed FMAVs ${ }^{[14-16]}$. In 2013, Harvard University developed the first sub-100 mg insect-scale FMAV which realized controlled flight and unconstrained stable hovering ${ }^{[17]}$.

Different from the piezoelectric actuation used in Harvard University and CMU's works, we presented the first liftoff of the world's smallest electromagnetically driven flapping-wing micro robot $^{[10]}$, indicating the electromagnetic actuators could successfully serve as the drive system for insect-inspired FMAVs. However, because only one electromagnetic actuator was designed to simultaneously drive two wings, this robot cannot produce body torques by asymmetric wing motion for maneuvering. Inspired by SCCPFM, we present a novel

\footnotetext{
*Corresponding author: Weiping Zhang

E-mail: zhangwp@sjtu.edu.cn
} 
electromagnetically driven insect-inspired tailless flapping-wing micro robot in this paper, as shown in Fig. 1. With the spherical four-bar mechanisms, two artificial wings are actuated by two electromagnetic actuators, respectively. With a total weight of $96 \mathrm{mg}$ and a wingspan of $35 \mathrm{~mm}$, this robot is capable of liftoff and all three control torques modulation.

\section{Design and fabrication}

\subsection{Overall design}

In order to solve the problem that the single electromagnetically driven robot (SJFly I) ${ }^{[10]}$ we presented previously is unable to generate control torques by asymmetric wing motion. The novel robot (SJFly II) presented in this paper utilizes two identical actuators to drive the two wings independently, thus asymmetrical wing motions are achieved as shown in Fig. 2, by which all three control torques (pitch, roll, and yaw) can be independently generated and modulated.

Specifically, the SJFly II is designed in an axially symmetric form. To avoid the electromagnetic interference, the two magnets of the actuators are fixed on the airframe (back to back) through two bosses to extend their distance, and each coil is respectively connected with the transmission as a drive unit in each side. Due to the back to back layout of the two actuators, the spherical four-bars are adopted in SJFly II to transform actuator vibrations into wing flapping movements referring to Refs. [14] and [15], and each coil of the actuators is respectively connected with each side the transmission as the drive unit. Excited by AC power sources, each coil produces reciprocating movements which mimics the contraction of the insect's back muscles, thereby driving the wing through the spherical four-bar the to generate periodic flapping motions. Meanwhile, the artificial wing generates thrust by its passive rotation that results from the inertia and aerodynamic forces.

\subsection{Optimized design of the electromagnetic actua- tor}

The performance of the electromagnetic actuator dominates the ability of the robot to drive the wing. SJFly II is developed based on SJFly I with the similar wingspan and weight. Thus, the total performance of the two novel actuators used in SJFly II should be close to the performance of the single actuator used in SJFly I. In this paper, the maximum electromagnetic force generated by the novel actuator is theoretically analyzed to evaluate its performance.

The structure of each electromagnetic actuator used in SJFly II consists of a neodymium iron boron magnet Ni-N52 fixed on the airframe and a copper coil stuck at the end of one side of the spherical four-bars. Since the actual output radian of the coil is small, it can be approximated as a linear displacement on the central axis of the magnet. When a current is applied into the coil, the electromagnetic reaction force acting on the coil can be calculated by:

$$
d \boldsymbol{F}_{e}=I d \boldsymbol{l} \times \boldsymbol{B},
$$

where $I d \boldsymbol{l}$ is the current element that instantaneously passes through the coil, and $\boldsymbol{B}$ is the magnetic induction at different positions of the coil relative to the magnet, as shown in Fig. 3a. However, due to the complexity of the magnetic field distribution, it is difficult to directly use Eq. (1) to calculate the electromagnetic reaction force. Thus, the magnet is simplified as a pair of magnetic dipoles in the analysis. Schematic diagram of the coil is shown in Fig. 3b, taking the midpoint of the coil as the coordinate origin $O$, then the magnitude of the magnetic
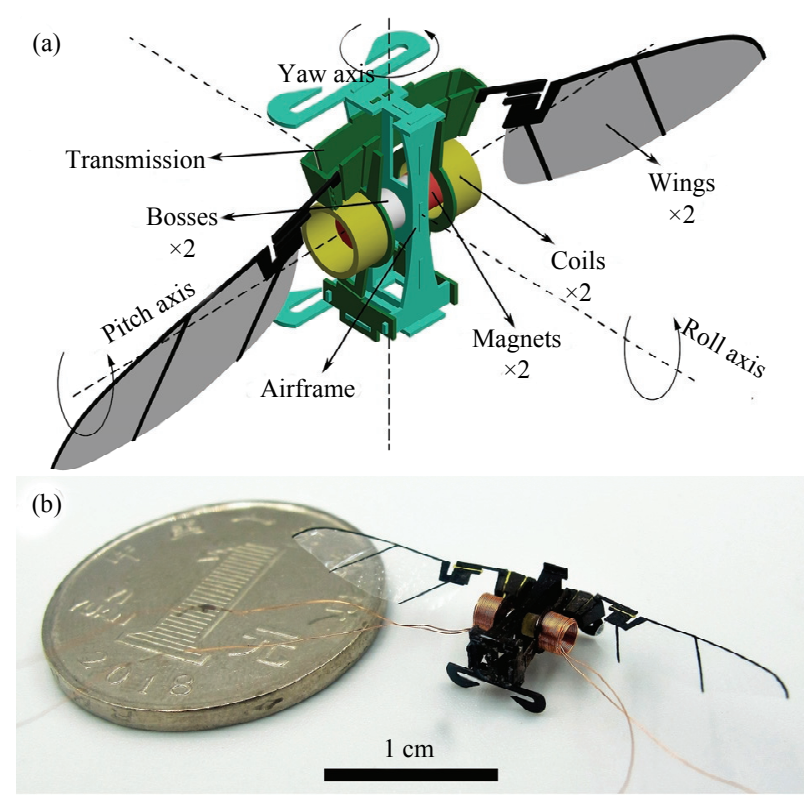

Fig. 1 (a) CAD model of the insect-inspired flapping-wing micro robot and (b) a prototype insect-inspired flapping-wing micro robot contrasted with a coin. 


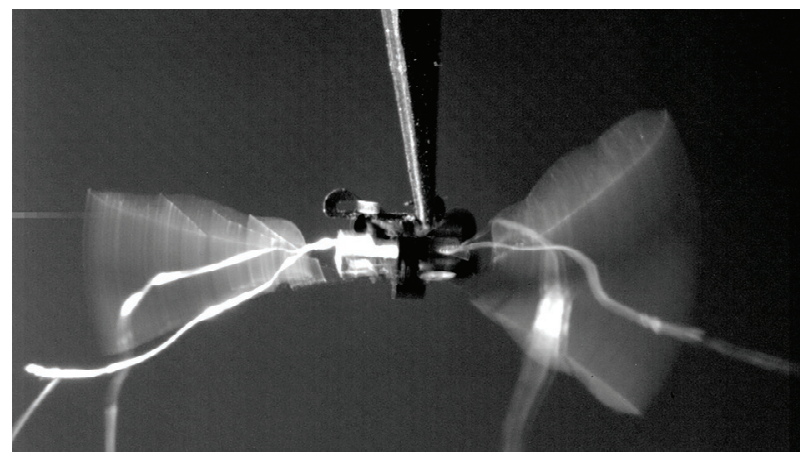

Fig. 2 Asymmetrical flapping of the two wings of the designed robot. The left wing is driven by a sinusoidal voltage with amplitude of $0.5 \mathrm{~V}$ and frequency of $90 \mathrm{~Hz}$, and the right wing is driven by a sinusoidal voltage with amplitude of $1 \mathrm{~V}$ and frequency of $90 \mathrm{~Hz}$.

induction at any point $P\left(x_{\mathrm{p}}, 0\right)$ on the axis can be calculated by:

$$
B_{\mathrm{p}, x}=\frac{\mu_{0} n_{1} n_{2} I}{2 L}\left(\cos \beta_{1}-\cos \beta_{2}\right),
$$

where $\mu_{0}$ is the vacuum permeability $\left(4 \pi \times 10^{-7} \mathrm{Tm} \cdot \mathrm{A}^{-1}\right)$, $L$ is the length of the coil, $I$ is the current passing through the coil, $n_{1}$ and $n_{2}$ are the number of turns of the coil in the length direction and the diameter direction, respectively. The values of $\cos \beta_{1}$ and $\cos \beta_{2}$ can be obtained by geometric relations:

$$
\left\{\begin{array}{l}
\cos \beta_{1}=\frac{x_{\mathrm{p}}+L / 2}{\sqrt{(D / 2)^{2}+\left(x_{\mathrm{p}}+L / 2\right)^{2}}} \\
\cos \beta_{1}=\frac{x_{p}+L / 2}{\sqrt{(D / 2)^{2}+\left(x_{\mathrm{p}}+L / 2\right)^{2}}}
\end{array}\right.
$$

where $D$ is the diameter of the coil. Assuming that the magnet (simplified as magnetic dipoles) is placed at point $P\left(x_{\mathrm{p}}, 0\right)$ and its magnetic moment is defined as $\boldsymbol{m}_{\mathrm{p}}$, then the electromagnetic reaction force acting on the coil can be calculated as:

$$
\boldsymbol{F}_{\mathrm{e}}=\nabla\left(\boldsymbol{m}_{\mathrm{p}} \cdot \boldsymbol{B}_{\mathrm{p}}\right) .
$$

Simplifying the magnet to a particle at point $P$, the magnitude of the electromagnetic reaction force in the $x$-axis direction is calculated as:

$$
F_{\mathrm{e}, x}=H_{\mathrm{c}} h \pi\left(\frac{d}{2}\right)^{2} \frac{d B_{\mathrm{p}, x}}{d x_{\mathrm{p}}},
$$

where $H_{\mathrm{c}}$ is the coercivity of the magnet, $d$ and $h$ are the diameter and height of the magnet respectively.

By the theoretical analysis above, the magnitude of the electromagnetic reaction force acting on the coil depends on the geometric and physical parameters of the coil and magnet, and the real-time current. Since the coil is easier to be customized than the magnet, the parameters of the magnet used in this paper are fixed as shown in Table 1, and then proper parameters of the coil are the key to the optimization of the electromagnetic force. To match the magnet, the diameter of the coil is fixed at $2.4 \mathrm{~mm}$ with the fixed number of turns in the diameter direction $\left(n_{2}\right)$ of 4 . The number of turns in the length direction $\left(n_{1}\right)$ is optimized to design the proper coil. The length of the coil $(L)$ is determined by $n_{1}$ and the wire diameter $\left(d_{\mathrm{w}}\right)$ as:

$$
L=k_{1} d_{\mathrm{w}} n_{1},
$$

where the $k_{1}$ is the correction factor due to the epoxy used to glue the wires (measured as 1.25), and $d_{\mathrm{w}}$ is $60 \mu \mathrm{m}$. Then, the current passing through the coil $(I)$ can be expressed as:

$$
I=\frac{U}{R_{1} n_{1}},
$$

where the $U$ is the input voltage of the electromagnetic actuator, and the $R_{1}$ is the resistance of one layer of coil (measured as $0.091 \Omega$ ).

Set $U=1.5 \mathrm{~V}$, the relationship of $F_{\mathrm{e}, x}, x_{\mathrm{p}}$ and $n_{1}$ is obtained by Eq. (5) as shown in Fig. 3c. It can be seen that as $n_{1}$ increases, the curves of the electromagnetic force gradually tend to be flat and delayed as shown in Fig. 3c, and the maximum electromagnetic force $\left(F_{\mathrm{e}, \max }\right)$ tends to decrease linearly as shown in Fig. 3d. In addition, an increase in $n_{1}$ also causes an increase in coil's weight, that is, a smaller $n_{1}$ of the coil is expected to achieve more optimized performance of the actuator.

However, if the length of the coil is too small, the reciprocating stroke of the actuator would be restricted, and linearity and reliability of the transmission would be reduced due to the excessive transmission ratio, which is not conducive for accurate control. Therefore, the limit position $\left(X_{\text {lim }}, 0\right)$ of reciprocating stroke of the actuator is introduced as defined in Fig. 3e. The limit position cannot be surpassed or the interference between the coil and the magnet might be caused, and $X_{\text {lim }}$ must meet the 


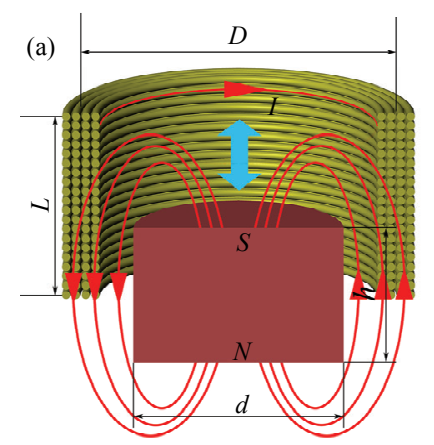

(c)

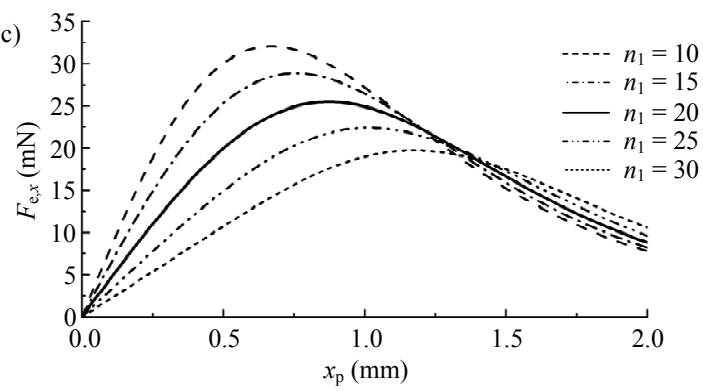

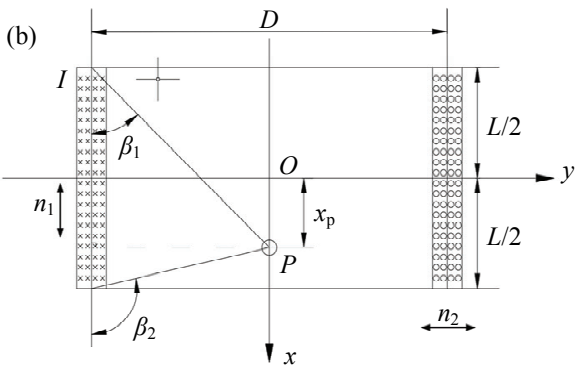
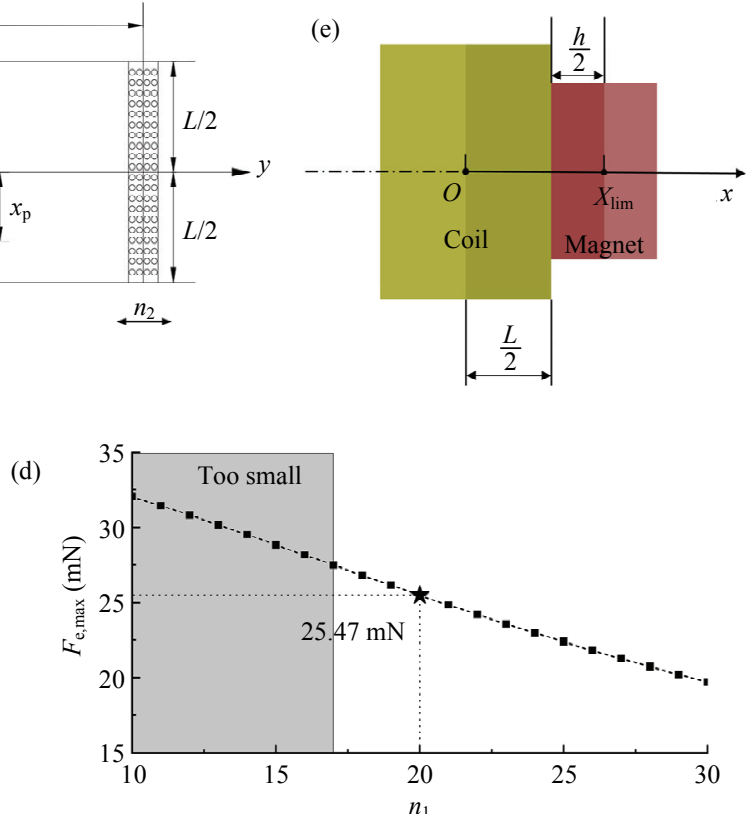

Fig. 3 (a) Section view of the electromagnetic actuator. (b) Schematic diagram of the coil. (c) The relationship between the electromagnetic force and the position of the coil relative to the magnet as $n_{1}$ increases from 10 to 30 (Only five curves are shown for simplicity). (d) The relationship between the maximum electromagnetic force and the $n_{1}$. (e) The limit position of reciprocating stroke of the actuator.

Table 1 Parameters of the magnet

\begin{tabular}{cc}
\hline Type & Ni-N52 \\
\hline Diameter $(\mathrm{mm})$ & 1.5 \\
Height $(\mathrm{mm})$ & 1 \\
Coercivity $\left(\mathrm{KA} \cdot \mathrm{m}^{-1}\right)$ & 800 \\
Mass $(\mathrm{mg})$ & 14 \\
\hline
\end{tabular}

following conditions:

$$
X_{\lim }=\frac{L}{2}+\frac{h}{2} \geq \frac{\varphi_{\max }}{2} / T_{d}
$$

where the $\varphi_{\max }$ is the maximum flapping angle (peak-to-peak) of the robot's wing (set as $130^{\circ}$ ), and the $T_{d}$ is the desired transmission ratio ( $\mathrm{set}$ as $1 \mathrm{rad} \cdot \mathrm{mm}^{-1}$ ), and then $n_{1} \geq 17$ is yielded by Eqs. (6) and (8).

In this paper, $n_{1}$ is chosen as 20 for safety margins. The adopted parameters of the coil are shown in Table 2. As shown in Fig. 3d, the maximum electromagnetic force generated by a novel actuator is estimated as $25.47 \mathrm{mN}$ when $x_{\mathrm{p}}$ is $\pm 0.87 \mathrm{~mm}$ (almost at both ends of the coil), close to half of the maximum electromagnetic force $(25 \mathrm{mN})$ generated by SJFly $\mathrm{I}^{[10]}$.

\subsection{Transmission}

To impedance-match the actuator to the load as well as amplify the motion of the actuator, spherical four-bar mechanisms are adopted as the transmission of the SJFly II with all joint axes meet at the center of the rotary axis of the actuator, as shown in Fig. 4a. The Smart Composite Manufacturing (SCM) process ${ }^{[18]}$ and the creation of SJFly I give us confidence in the precise fabrication and assembly of these micro mechanical components. The transmission is made from two rigid structural layers of $80 \mu \mathrm{m}$ thick carbon fiber and a flexible deformable layer of $7.5 \mu \mathrm{m}$ thick polyimide film sandwiching between them. As shown in Fig. 4b, the transmission is shape by UV laser in two-dimension, and then folded and glued into three-dimension. The transmission ratio is measured as $1.196 \mathrm{rad} \cdot \mathrm{mm}^{-1}$ due to errors caused by the flexible hinges.

\subsection{Wings}

Several insect-inspired artificial wings have been reported $^{[19-23]}$, and the artificial wing used in this paper is designed to imitate the wing of drone-fly Eristalis tenax in similar size and shape ${ }^{[24]}$. The artificial wing is made from $1.5 \mu \mathrm{m}$ thick polyester as the membrane and $60 \mu \mathrm{m}$ thick carbon fiber as the veins and root to realize high modulus and light weight. By the non-rigid 
Table 2 Parameters of the coil

\begin{tabular}{cc}
\hline Parameters & Value \\
\hline Diameter $(\mathrm{mm})$ & 2.4 \\
Length $(\mathrm{mm})$ & 1.5 \\
Wire diameter $(\mu \mathrm{m})$ & 60 \\
Number of turns in length direction & 20 \\
Number of turns in diameter direction & 4 \\
Mass $(\mathrm{mg})$ & 16 \\
\hline
\end{tabular}

connection of the flexible hinge made of $7.5 \mu \mathrm{m}$ thick polyimide on the wing root, the wing will produce passive rotations under aerodynamic moment during the reciprocating beat, which is the main factor for the generation of lift force. Compared to the wing used in SJFly I, this artificial wing is optimized with thinner veins and a smaller wing root to further reduce the inertia of the wing, as shown in Fig. 4c.

\subsection{Airframe}

The airframe used in SJFly II is shown in Fig. 4d. Each component of the airframe is shape by UV laser in two-dimension, and then assembled into three-dimension. To be noticed, these components are made from two layers of $80 \mu \mathrm{m}$ thick carbon fiber with fiber directions orthogonal to reduce the weight as well as enhance the strength.

As a result, distribution of the total mass of the SJFly II is shown in Fig. 5, and the two electromagnetic actuators (including double coils, magnets and bosses) account for almost $2 / 3$ of the total weight.

\section{Experiments}

\subsection{Lift measurement and liffoff}

Since the lift produced by the insect-inspired flapping-wing micro robot presented in this paper is only in $\mathrm{mN}$-scale that cannot be directly measured by all commercial force sensors, a customized lift test platform is designed to measure the real-time lift of the robot and other insect-sized FMAV referring to Ref. [25] in this paper, as shown in Fig. 6a. The Invar-made doublecantilever beam transforms the lift produced by the robot into slight parallel displacement along measurement direction of a capacitive displacement sensor (capaNCDT6530, MICRO-EPSILON), thus the real-time lift can be derived by measuring the displacement of the target plate. Calibrated by standard weights, the lift test platform has a sensitivity of $2.1866 \mu \mathrm{m} \cdot \mathrm{mN}^{-1}$, a dynamic resolution of $0.457 \mu \mathrm{N}(0.0466 \mathrm{mg})$. In addition, the measurement uncertainty are mainly induced by the fitting error of calibration $(0.02612 \mathrm{mN})$, the indication error of standard weights $(0.0002686 \mathrm{mN})$, and the absolute error of the capacitive displacement sensor $(0.05388 \mathrm{mN})$. As a results, the uncertainty of the lift test platform is estimated as $0.02694 \mathrm{mN}$. The detailed design and uncertainty analysis of the lift test platform are shown in Online Resource 1. In order to avoid the interaction between the Invar structure and the magnets of the robot, a long carbon fiber truss is introduced to extend the distance between the robot and the support plate, as shown in Fig. 6 .

Based on the Blade Element Theory (BET) ${ }^{[16,26]}$, the mean lift generated by single wing can be expressed as:

$$
F_{\mathrm{L}, \text { single }}=\frac{1}{4} \rho_{\text {air }} \bar{C}_{\mathrm{L}} R^{3} \bar{c}_{2}^{2}(2 \pi f)^{2} \cdot \Psi^{2},
$$

where $\rho_{\text {air }}$ is the density of air $\left(1.29 \mathrm{Kg} \cdot \mathrm{m}^{-3}\right), \bar{C}_{\mathrm{L}}$ is the mean lift coefficient (1.8), $R$ is the wing length $(13 \mathrm{~mm})$, $\bar{c}$ is the mean chord length $(3.8 \mathrm{~mm}), \hat{r}_{2}^{2}$ is the $2 n d$ dimensionless moments of wing area (0.292) defined in Ref. [24], $f$ and $\Psi$ are the flapping frequency and amplitude of the wing respectively. When $f$ is fixed, the flapping amplitude of the wing is almost proportional to the amplitude of the input sinusoidal voltage as $\Psi=k_{2} V_{\text {amp }}{ }^{[15]}$, the scale factor $k_{2}$ is measured as $0.814 \mathrm{rad} \cdot \mathrm{V}^{-1}$ for this robot. Applied the same sinusoidal voltage to the two actuators, the total lift generated by the robot is yielded:

$$
F_{\mathrm{L}, \text { tot }}=2 F_{\mathrm{L}, \text { single }}=2 \rho_{\mathrm{air}} \bar{C}_{\mathrm{L}} R^{3} \bar{c} \hat{r}_{2}^{2} f^{2} k_{2}^{2} \cdot V_{\mathrm{amp}}^{2} .
$$

Thus the lift generated by the robot can be modulated by the amplitude of the input sinusoidal voltage. A sinusoidal voltage with fixed $f$ of $80 \mathrm{~Hz}$ is applied to both of the two actuators. The calculated and measured relationships between the mean lift of the robot and the amplitude of the input sinusoidal voltage are shown in Fig. 7a. It shows that the measured mean lift generated by the robot is proportional to the square of the input voltage amplitude, close to the calculated mean lift with a small deviation. The generated lift is slightly underestimated by the aerodynamic model, because 


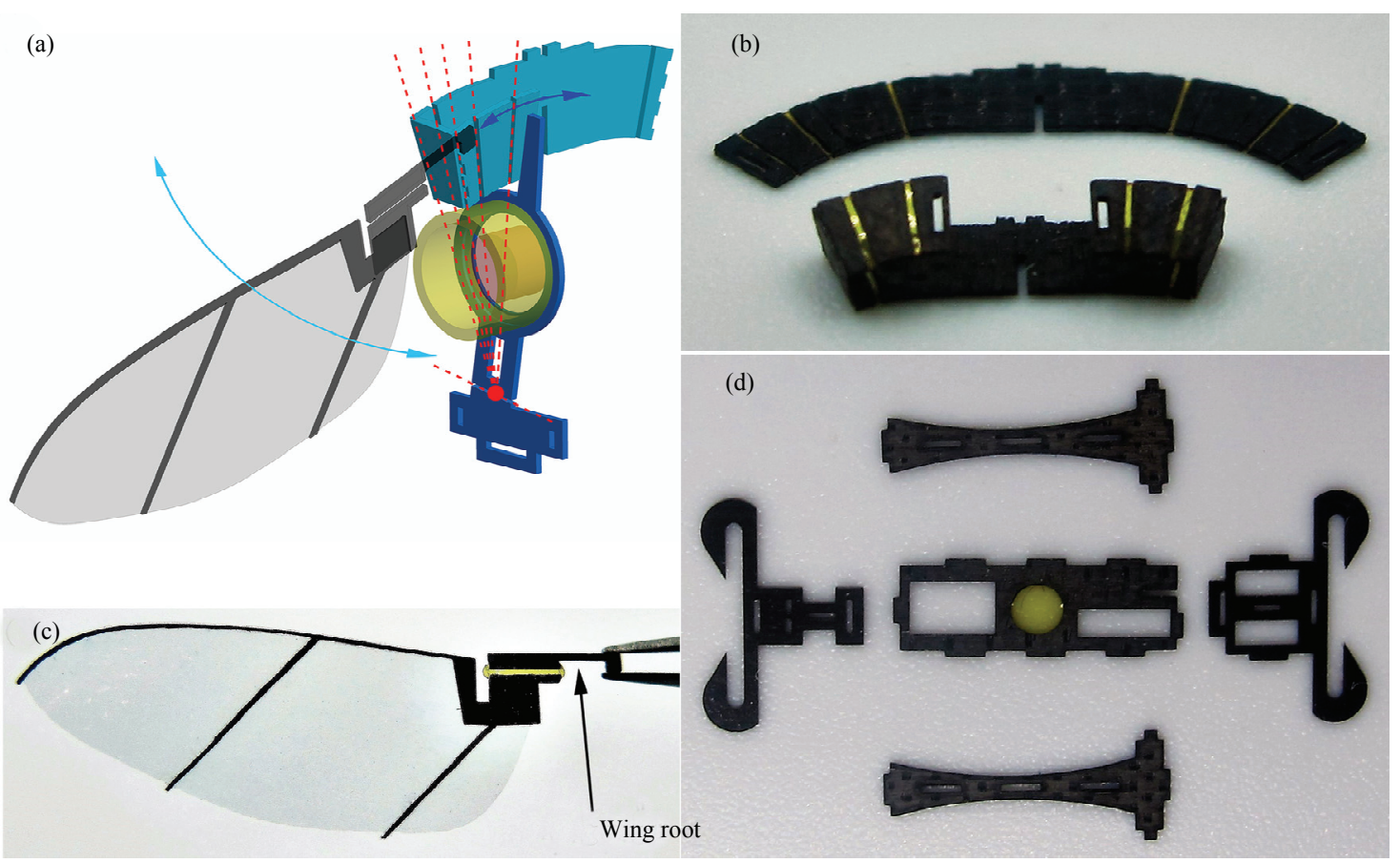

Fig. 4 (a) Schematic diagram of the spherical four-bar transmission in the SJFly II. Notice that joint axes of the transmission meet at the center of the rotary axis of the electromagnetic actuator. Photos of (b) the transmission before and after folding, (c) the left artificial wing, and (d) separate components of the airframe.

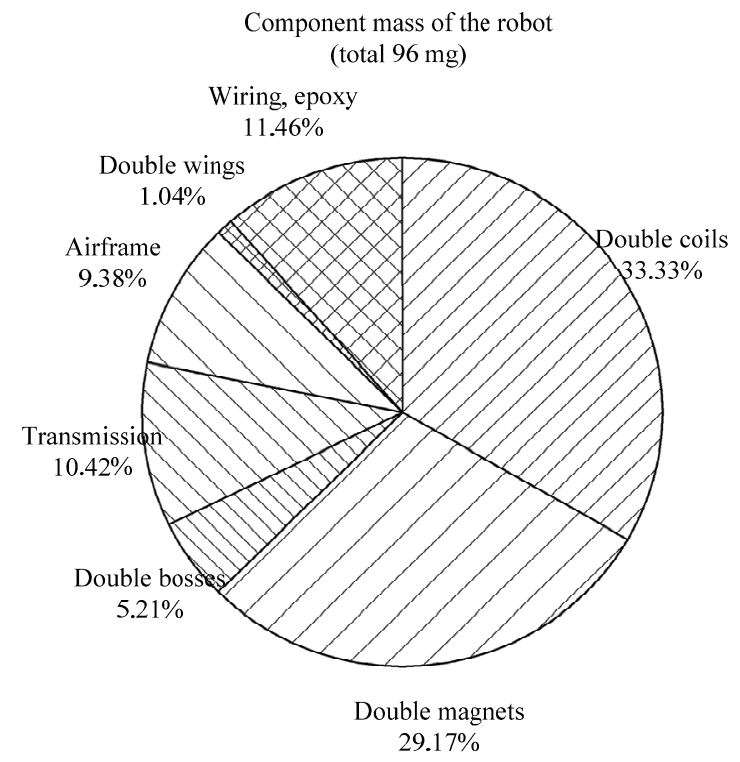

Fig. 5 Distribution of the total FMAV mass.

some complex unsteady aerodynamic mechanisms are ignored. However, when $V_{\text {amp }}$ is greater than $1.4 \mathrm{~V}$, the increase in the measured mean lift slows down and tends to saturation, because the movement of the transmission is close to its limit position at this time, limiting the further increase of the flapping angles. The real-time lift during two flapping cycles at $V_{\text {amp }}$ of $1.5 \mathrm{~V}$ is shown in Fig. 7b. It shows that effective lift is mainly generated in the middle of each stroke, and the lift at each stroke reversal is small or even negative. Ideally, the lift curve during the upstroke and downstroke should be symmetric. However, in this case, asymmetry of the lift is mainly caused by the asymmetry of robot structure induced by the machining and assembly errors.

When $V_{\text {amp }}$ is greater than approximately $1.3 \mathrm{~V}$, the mean lift generated by the robot exceeds its own weight $(0.94 \mathrm{mN})$, as shown in Fig. 7a. The same experimental setup in Ref. [9] is used to demonstrate the liftoff of the robot. Captured by a high-speed camera (Phantom LC111) at 3000 frames per second, the robot moves along the guide rail vertically when a sinusoidal voltage with frequency of $80 \mathrm{~Hz}$ and amplitude of $1.5 \mathrm{~V}$ is applied, as shown in Fig. 8.

\subsection{Control torques measurement}

The three rotation axes of roll, pitch, and yaw are defined in Fig. 1a. Similar with the lift measurement, a customized torque test platform is designed for the 

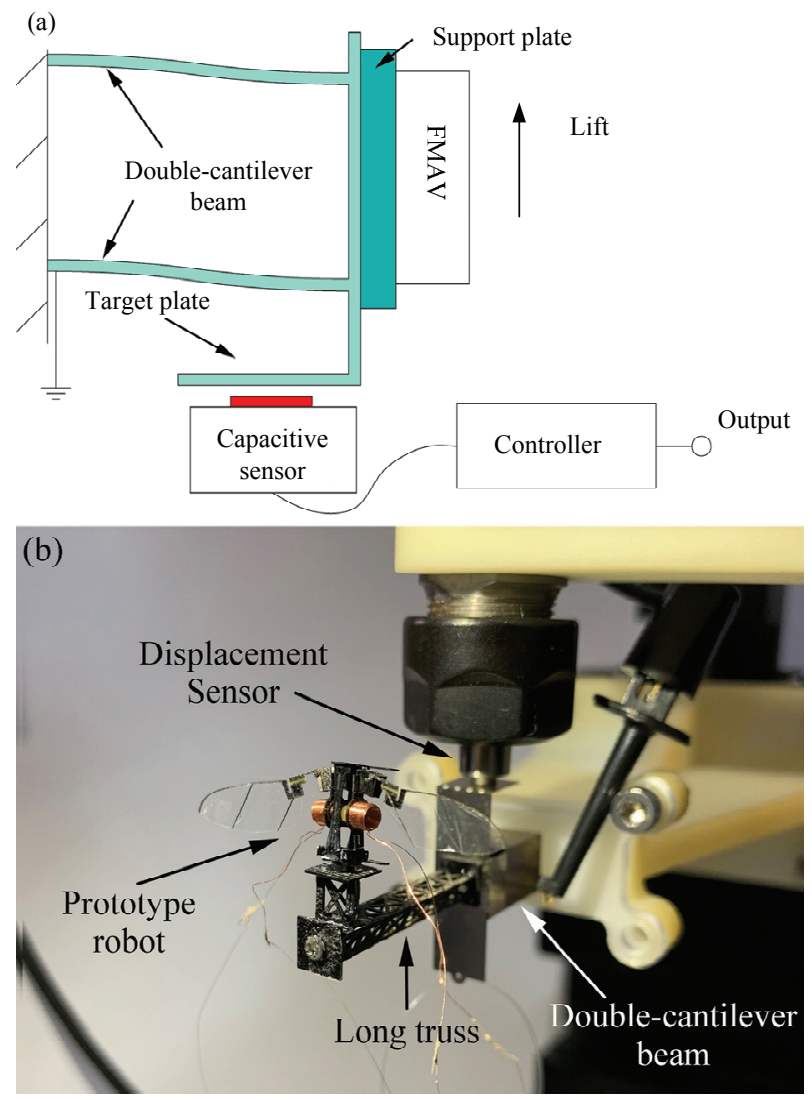

Fig. 6 (a) Schematic of the customized lift test platform. (b) The robot mounted on the customized lift test platform through a long truss.

three torques measurements referring to Ref. [27]. Its test schematic is shown in Fig. 9a. The Invar-made cross-shaped beam transforms the torque about the central axis of the beam into slight rotation of the target plate, which can be regarded as parallel displacement. Thus, the real-time torque can be derived by measuring the displacement with the capacitive displacement sensor (capaNCDT6530, MICRO-EPSILON). After calibration, the torque test platform has a sensitivity of $0.09027 \mu \mathrm{m} \cdot \mu \mathrm{Nm}^{-1}$, a dynamic resolution of $0.0111 \mu \mathrm{Nm}$. Similar to the lift test platform, the measurement uncertainty are also mainly induced by the fitting error of calibration $(0.20333 \mu \mathrm{Nm})$, errors of standard input torque $(0.01155 \mu \mathrm{Nm})$, and the absolute error of the capacitive displacement sensor $(0.27695 \mu \mathrm{Nm})$. Then, the uncertainty of the torque test platform is $0.25893 \mu \mathrm{Nm}$. The detailed design and uncertainty analysis of the torque test platform are shown in Supplementary file. The setup of the control torques measurement is shown in Fig. 9b, in the case, the pitch torque is measured. For the roll and yaw torques measurement, the robot needs to be remounted to make its specific axis of rotation parallel to the sensing direction of the system, as shown in Figs. 9c and 9d.

\subsubsection{Roll torque}

The roll torque $\tau_{\text {roll }}$ is generated by the difference in the magnitude of the mean lift generated by the left and right wings $\left(F_{\mathrm{L}, \text { left }}, F_{\mathrm{L}, \text { right }}\right)$, as shown in Fig. 10a.

$$
\tau_{\text {roll }}=r_{\mathrm{cp}}\left(F_{\mathrm{L}, \text { left }}-F_{\mathrm{L}, \text { right }}\right),
$$

where $r_{\mathrm{cp}}$ is the radius of the pressure center of the wing $(10.3 \mathrm{~mm}), V_{\text {amp,left }}$ and $V_{\text {amp,right }}$ are the amplitude of the two input sinusoidal voltages respectively and determined by basic amplitude $V_{\text {amp,basic }}$ and amplitude difference $V_{\text {diff }}$ as:

$$
\left\{\begin{array}{l}
V_{\text {amp,left }}=V_{\text {amp,basic }}+V_{\text {diff }} \\
V_{\text {amp,right }}=V_{\text {amp,basic }}-V_{\text {diff }}
\end{array} .\right.
$$

By substituting Eqs. (9) and (12) into Eq. (11), $\tau_{\text {roll }}$ is proportional to $V_{\text {amp,basic }}$ and $V_{\text {diff }}$ as:

$$
\tau_{\text {roll }}=4 r_{\text {cp }} \rho_{\text {air }} \bar{C}_{\mathrm{L}} R^{3} \bar{c} \hat{r}_{2}^{2} f^{2} k_{2}^{2} V_{\text {amp,basic }} \cdot V_{\text {diff }} \cdot
$$

Two sinusoidal voltages with fixed $f$ of $80 \mathrm{~Hz}$ and $V_{\text {amp,basic }}$ of $1 \mathrm{~V}$ are applied to both of the two electromagnetic actuators. By adjusting $V_{\text {diff }}$ of the two input sinusoidal voltages, the real-time roll torque generated by the robot is measured by the torque test platform. The calculated and measured relationships between the mean roll torque and $V_{\text {diff }}$ are shown in Fig. 11a. Close to the calculated result, the measured roll torque is highly proportional to the amplitude difference.

\subsubsection{Pitch torque}

The pitch torque $\tau_{\text {pitch }}$ is generated by the offset between the center of the total lift and the gravity of the robot, and the offset can be modulating by shifting the mean stroke angle $\theta$, as shown in Fig. $10 \mathrm{~b}$.

$$
\tau_{\text {pitch }}=r_{\mathrm{cp}} F_{\mathrm{L}, \mathrm{tot}} \sin \theta
$$

The mean stroke angle $\theta$ of the wing is approximately proportional to the DC offset $V_{\text {off }}$ of the input voltage as $\theta=k_{3} V_{\text {off }}{ }^{[15]}$, the scale factor $k_{3}$ is measured 

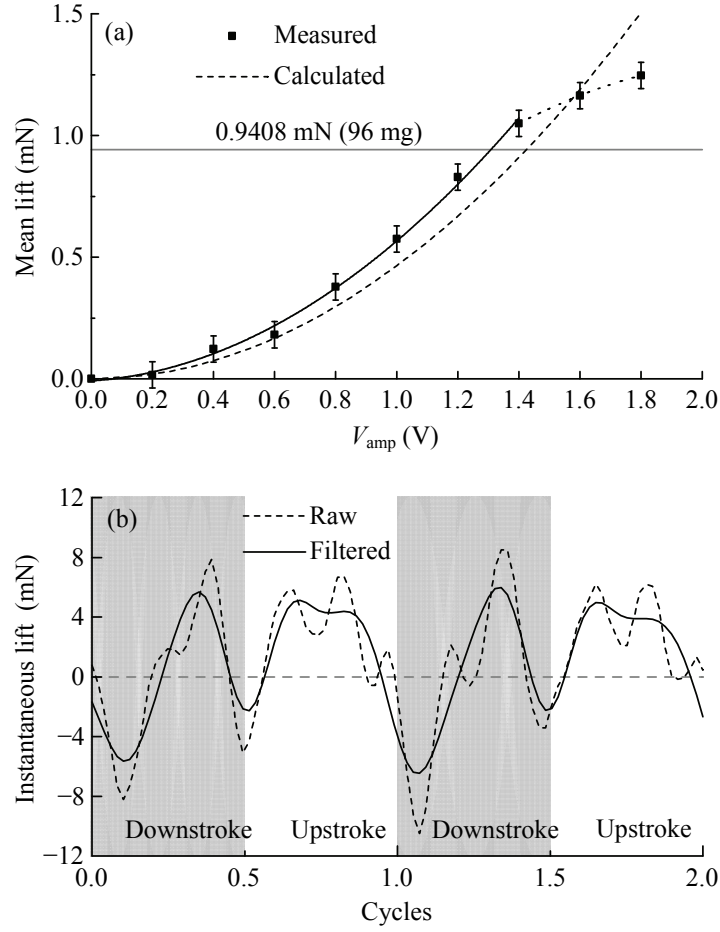

Fig. 7 (a) The calculated and measured relationships between the mean lift of the robot and the amplitude of the input sinusoidal voltage. The solid line and the dotted line are the quadratic fit of the measured results from 0 to $1.4 \mathrm{~V}$ and $1.4 \mathrm{~V}$ to $1.8 \mathrm{~V}$ respectively. Each measured point in the graph is averaged over one hundred cycles of filtered real-time lift. (b) The measured real-time lift curve during two flapping cycles, the raw lift data is filtered by a digital low pass filter with cutoff frequency of $200 \mathrm{~Hz}$ to reduce the interference from high-frequency mechanical and electrical noise.

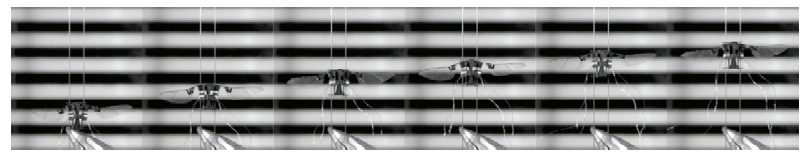

Fig. 8 Liffoff of the insect-inspired flapping-wing micro robot, the interval between the images is approximately $200 \mathrm{~ms}$.

as $1.35 \mathrm{rad} \cdot \mathrm{V}^{-1}$ for this robot. Thus, when the $\theta$ is small $(\theta \approx \sin \theta), \tau_{\text {pitch }}$ can be expressed as:

$$
\tau_{\text {pitch }}=2 r_{\text {cp }} \rho_{\text {air }} \bar{C}_{\mathrm{L}} R^{3} \bar{c}_{2}^{2} f^{2} k_{2}^{2} k_{3} V_{\text {amp }}^{2} \cdot V_{\text {off }} .
$$

A sinusoidal voltage with fixed $f$ of $80 \mathrm{~Hz}$ and $V_{\text {amp }}$ of $1 \mathrm{~V}$ is applied to both of the two actuators. By adjusting $V_{\text {off }}$ of the input sinusoidal voltage, the pitch torque generated by the robot is measured. As shown in Fig. $11 \mathrm{~b}$, the mean measured roll torque is highly proportional to the DC offset, which is close to the calculated result.

\subsubsection{Yaw torque}

The yaw torque $\tau_{\text {yaw }}$ is generated by the difference in the drag force acting on the left and right wings: the drag force acting on the faster flapping wing is larger than the slower in a half flapping stroke. In this paper, the modulation of the yaw torque is achieved by $\mathrm{SCCPFM}^{[13]}$, as shown in Fig. $17 \mathrm{c}$, the control signal in a complete period is composed of two half-cycle sinusoidal signals with different frequencies, the frequency of the upstroke control signal of the right wing is higher than that of the downstroke, resulting in the upstroke flapping velocity of controlled right wing faster than the downstroke, which is the opposite of the left wing controlled by the complementary control signal. Since the fundamental frequency of the control signal in a complete period does not change, the continuous yaw torque in the same direction is generated since the faster half stroke and the slower half stroke of the two wings are periodically alternated. The split factor $\delta$ is introduced in Fig. 10c to define how "splitting" the control signal is, and $\delta=0.5$ represents a standard sinusoid signal. Refer to Refs. [15, 16], the relationship between the mean drag force $F_{\mathrm{D}}$ acting on the wing and the split factor $\delta$ can be approximately as:

$$
F_{\mathrm{D}}=\frac{1}{4} \rho_{\mathrm{air}} \bar{C}_{\mathrm{D}} R^{3} \bar{c}_{2}^{2} \pi^{2} f^{2} \Psi^{2} \cdot\left(\frac{1-2 \delta}{\delta-\delta^{2}}\right),
$$

where $\bar{C}_{\mathrm{D}}$ is the mean drag coefficient (1.9). Since the two wings are controlled by two complementary signals, the mean drag force acting on the two wings is determined:

$$
F_{\mathrm{D}, \text { left }}=-F_{\mathrm{D}, \text { right }} .
$$

Thus, for $\delta \in(0,1)$, the $\tau_{\text {yaw }}$ can be expressed as:

$$
\begin{aligned}
\tau_{\text {yaw }} & =-r_{\text {cp }}\left(F_{\mathrm{D}, \text { left }}-F_{\text {D,right }}\right) \\
& \approx 4 r_{\text {cp }} \rho_{\text {air }} \bar{C}_{D} R^{3} \hat{c}_{2}^{2} f^{2} k_{2}^{2} V_{\text {amp }}^{2} \cdot(\delta-0.5) .
\end{aligned}
$$

In this paper, two complementary SCCPFM voltages both with fixed frequency of $80 \mathrm{~Hz}$ and fixed amplitude of $1 \mathrm{~V}$ are respectively applied to the two actuators. By adjusting $\delta$, the yaw torque generated by the robot is measured. The relationship between the mean measured yaw torque and the split factor $\delta$ is close to the calculated result, as shown in Fig. 11c. 
(a)
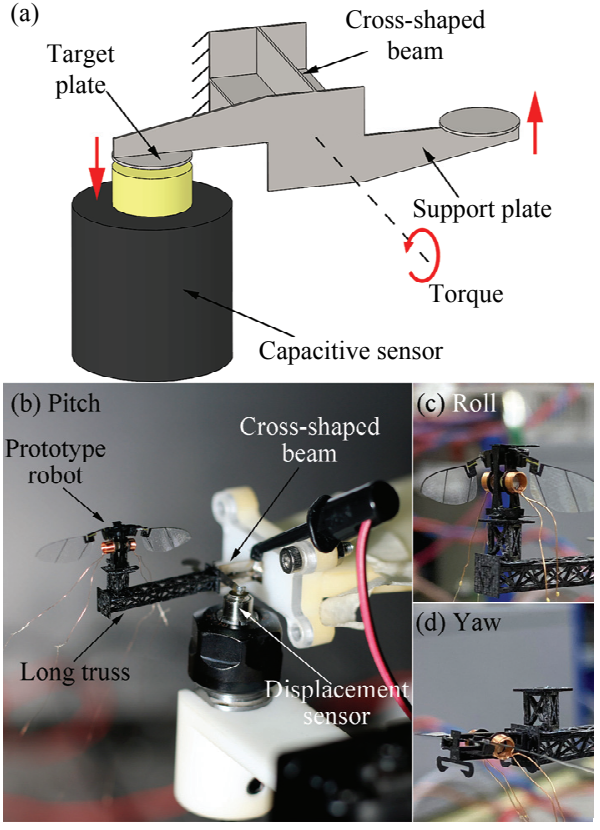

Fig. 9 (a) Schematic of the customized torque test platform. (b) The setup of the pitch torque measurement. Remounting for (c) roll and (d) yaw torque measurement.

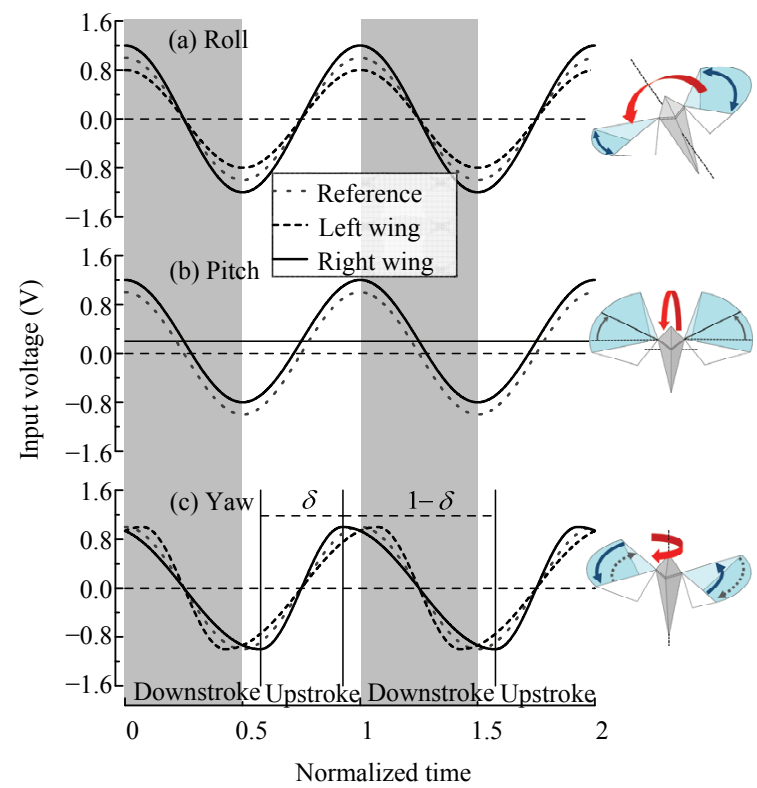

Fig. 10 Example diagram of the mechanism of roll, pitch, and yaw torque. (a) The difference in driving voltage amplitude of the left and right wings causes the mean lift generated by the two wings to be different, then the roll torque is generated. (b) The flapping plane of the two wings is shifted when the driving voltage of the two wings is raised, thus the pitch torque is generated by the offset between the lift vector and the center of the gravity of the robot. (c) One cycle of sinusoidal signal of the right wing is split into two half-cycle sinusoidal signals with different frequencies, the split factor $\delta$ is introduced to define how "splitting" the signal is, and the control signals of the left and right wings are complementary symmetrical.
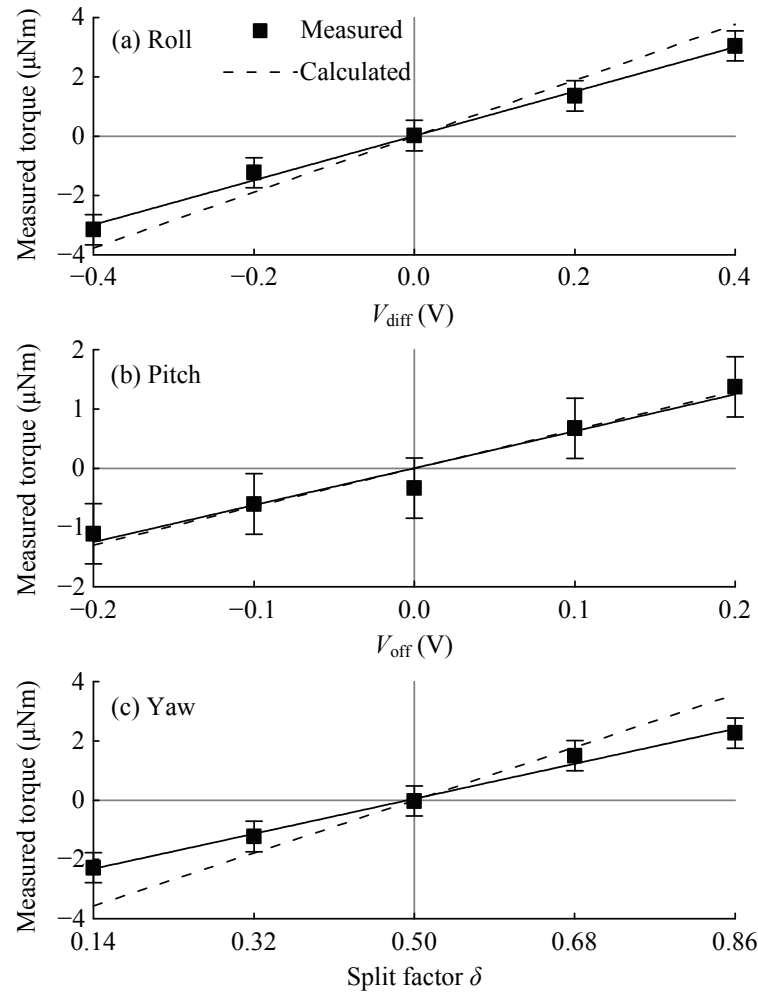

Fig. 11 Each measured torque is almost proportional to its control variable. Each measured point is averaged over one hundred cycles of filtered real-time torque, and the three solid lines are the linear fit of the measured points.

\section{Discussion and conclusion}

In this paper, we have demonstrated the design of a novel sub $100 \mathrm{mg}$ electromagnetically driven flapping-wing micro robot. Compared with the self-lifting insect-inspired flapping-wing robot we presented previously ${ }^{[10]}$ which utilizes only one electromagnetic actuator to generate symmetrical flapping of the two wings and therefore cannot achieve steering, this robot utilizes two optimized electromagnetic actuators placed back to back to drive the two wings independently though the spherical four-bar transmissions, thus this robot can generate all three torques of pitch, roll, and yaw for steering by asymmetrical flapping of the two wings, making it possible to achieve further controlled flight.

The novel electromagnetic actuator is optimized theoretically by determining the appropriate parameter $\left(n_{1}\right)$ of the coil. As a result, the total maximum electromagnetic force of two novel electromagnetic actuators is estimated as $50.94 \mathrm{mN}$, close to the maximum electro- 
magnetic force of $50 \mathrm{mN}$ generated by the robot SJFly I we presented previously ${ }^{[9]}$, which is also sufficient to drive the novel robot.

The ability of the robot to generate and modulate the lift and control torques is what we are most concerned about. Since the lift and torques generated by this insect-scale robot are too small to be measured directly by commercial force/torque sensors at present, customized lift and torque test platforms are developed to meet the measurement needs of the robot, and their design details and uncertainly analysis are shown in Supplementary file. In addition, a simplified aerodynamic model is built to estimate the mean lift as well as the three torques. As predicted by the aerodynamic model, the measured mean lift generated by the robot is proportional to the square of the input voltage amplitude and fits well with the calculated mean lift when the transmission is not close to the limit position, and the mean lift generated by the robot exceeds its own weight when the input voltage amplitude is greater than $1.3 \mathrm{~V}$. This robot successfully liftoff along the guide rail vertically when inputting a sinusoidal voltage with an amplitude of 1.5 V. Furthermore, based on the aerodynamic model, the mean torques of roll, pitch and yaw are proportional to the amplitude difference, the DC offset, and the introduced split factor $\delta$ of the two input signals, respectively, which is also verified by the measured results with good match. To be noticed, the three control parameters of torques are decoupled from each other, that is, each control torque of the robot can be independently modulated by its specific control parameter, which is helpful for the further controlled flight.

From above, the insect-inspired flapping-wing micro robot presented in this paper can generate sufficient lift to take off and modulate all three control torques for steering, indicating that the electromagnetic actuation can be successfully served as the driving system for the sub-100 mg flapping-wing robots. To achieve controlled flight of the robot, some work is needed in the future. Aerodynamics of the flapping flight and system dynamics of the robot should be modeled in more detail. Robust control algorithm based on external motion capture should be studied. Moreover, micro power source, micro sensors, and optimization of the flight efficiency are worth exploring to achieve the final un- tethered flight of the robot.

\section{Acknowledgment}

This research was supported by the Supporting Foundation of the Ministry of Education of the People's Republic of China (6141A02022607, 6141A02022627), Shanghai Science and Technology Commission Project (19511104202), Shanghai Professional technical service platform (19DZ2291103), and the Pre-research Fund (17070107).

* All supplementary materials are available at https://doi.org/10.1007/s42235-020-0103-7.

Open Access This article is licensed under a Creative Commons Attribution 4.0 International License, which permits use, sharing, adaptation, distribution and reproduction in any medium or format, as long as you give appropriate credit to the original author(s) and the source, provide a link to the Creative Commons licence, and indicate if changes were made.

The images or other third party material in this article are included in the article's Creative Commons licence, unless indicated otherwise in a credit line to the material. If material is not included in the article's Creative Commons licence and your intended use is not permitted by statutory regulation or exceeds the permitted use, you will need to obtain permission directly from the copyright holder.

To view a copy of this licence, visit http://creativecommons.org/licenses/by/4.0/.

\section{References}

[1] Tu Z, Fei F, Deng X Y. Untethered flight of an at-scale dual-motor hummingbird robot with bio-inspired decoupled wings. IEEE International Conference on Robotics and Automation, Paris, France, 2020.

[2] Karasek M, Muijres F T, De Wagter C, Remes B D, De Croon G C. A tailless aerial robotic flapper reveals that flies use torque coupling in rapid banked turns. Science, 2018, 361, 1089-1094.

[3] Roshanbin A, Altartouri H, Karasek M, Preumont A. COLIBRI: A hovering flapping twin-wing robot. International Journal of Micro Air Vehicles, 2017, 9, 270-282.

[4] Phan H V, Kang T, Park H C. Design and stable flight of a 21 $\mathrm{g}$ insect-like tailless flapping wing micro air vehicle with angular rates feedback control. Bioinspiration \& Biomimet- 
ics, 2017, 12, 036006.

[5] Phan H V, Nguyen Q V, Truong Q T, Van Truong T, Park H C, Goo N S, Byun D, Kim M J. Stable vertical takeoff of an insect-mimicking flapping-wing system without guide implementing inherent pitching stability. Journal of Bionic Engineering, 2012, 9, 391-401.

[6] Keennon M, Klingebiel K, Won H. Development of the nano hummingbird: A tailless flapping wing micro air vehicle. 50th AIAA Aerospace Sciences Meeting including the New Horizons Forum and Aerospace Exposition, Nashville, USA, 2012, 0588.

[7] Au L T K, Park H C. Influence of center of gravity location on flight dynamic stability in a hovering tailless FW-MAV: Lateral motion. Journal of Bionic Engineering, 2020, 17, $148-160$.

[8] Wood R J. The first takeoff of a biologically inspired at-scale robotic insect. IEEE Transactions on Robotics, 2008, 24, 341-347.

[9] Roll J A, Cheng B, Deng X Y. An electromagnetic actuator for high-frequency flapping-wing microair vehicles. IEEE Transactions on Robotics, 2015, 31, 400-414.

[10] Zou Y, Zhang W P, Zhang Z. Liftoff of an electromagnetically driven insect-inspired flapping-wing robot. IEEE Transactions on Robotics, 2016, 32, 1285-1289.

[11] Zou Y, Zhang W P, Ke X J, Lou X L, Zhou S. The design and microfabrication of a sub $100 \mathrm{mg}$ insect-scale flapping-wing robot. Micro \& Nano Letters, 2017, 12, 297-300.

[12] Zou Y, Zhang W P, Zhou S, Ke X J, Cui F, Liu W. Monolithic fabrication of an insect-scale self-lifting flapping-wing robot. Micro \& Nano Letters, 2018, 13, 267-269.

[13] Oppenheimer M W, Doman D B, Sigthorsson D O. Dynamics and control of a biomimetic vehicle using biased wingbeat forcing functions. Journal of Guidance, Control, and Dynamics, 2011, 34, 204-217.

[14] Hines L L, Arabagi V, Sitti M. Free flight simulations and pitch and roll control experiments of a sub-gram flapping-flight micro aerial vehicle. IEEE International Conference on Robotics and Automation, Shanghai, China, 2011, $1-7$.

[15] Ma K Y, Felton S M, Wood R J. Design, fabrication, and modeling of the split actuator microrobotic bee. IEEE/RSJ International Conference on Intelligent Robots \& Systems,
Vilamoura, Portugal, 2012, 1133-1140.

[16] Zhang J, Cheng B, Deng X Y. Instantaneous wing kinematics tracking and force control of a high-frequency flapping wing insect MAV. Journal of Micro-Bio Robotics, 2016, 11, 67-84.

[17] Ma K Y, Chirarattananon P, Fuller S B, Wood R J. Controlled flight of a biologically inspired, insect-scale robot. Science, 2013, 340, 603-607.

[18] Wood R J, Avadhanula S, Sahai R, Steltz E, Fearing R S. Microrobot design using fiber reinforced composites. Journal of Mechanical Design, 2008, 130, 052304.

[19] Shang J K, Combes S A, Finio B M, Wood R J. Artificial insect wings of diverse morphology for flapping-wing micro air vehicles. Bioinspiration \& Biomimetics, 2009, 4, 036002.

[20] Tanaka H, Wood R J. Fabrication of corrugated artificial insect wings using laser micromachined molds. Journal of Micromechanics and Microengineering, 2010, 20, 075008.

[21] Bao X Q, Bontemps A, Grondel S, Cattan E. Design and fabrication of insect-inspired composite wings for MAV application using MEMS technology. Journal of Micromechanics and Microengineering, 2011, 21, 125020.

[22] Phan H V, Park H C. Design and evaluation of a deformable wing configuration for economical hovering flight of an insect-like tailless flying robot. Bioinspiration \& Biomimetics, 2018, 13, 036009.

[23] Kumar D, Mohite P M, Kamle S. Dragonfly inspired nanocomposite flapping wing for micro air vehicles. Journal of Bionic Engineering, 2019, 16, 894-903.

[24] Ellington C P. The aerodynamics of hovering insect flight. II. Morphological parameters. Philosophical Transactions of the Royal Society B, 1984, 305, 17-40.

[25] Wood R J, Cho K J, Hoffman K. A novel multi-axis force sensor for microrobotics applications. Smart Materials and Structures, 2009, 18, 125002.

[26] Whitney J P, Wood R J. Conceptual design of flapping-wing micro air vehicles. Bioinspiration \& Biomimetics, 2012, 7, 036001.

[27] Finio B M, Galloway K C, Wood R J. An ultra-high precision, high bandwidth torque sensor for microrobotics applications. IEEE/RSJ International Conference on Intelligent Robots \& Systems, San Francisco, USA, 2011, 31-38. 